Supporting Information for

\title{
Changes in Surface Area and Concentrations of Semivolatile Organic Contaminants in Ageing Snow
}

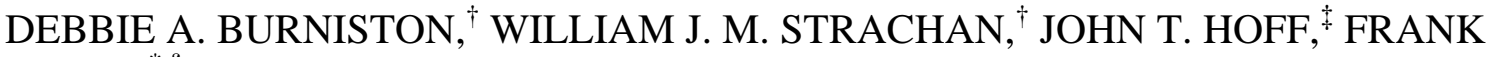 \\ WANIA $^{*}, \S$ \\ ${ }^{\dagger}$ Aquatic Ecosystem Protection Research Division, Environment Canada, 867 Lakeshore Road, \\ Burlington, Ontario, Canada L7R 4A6. \\ ‡ 15 Mohegan Crescent, London, Ontario, Canada N5V 2X7 \\ $\S$ Department of Physical and Environmental Sciences, University of Toronto Scarborough, 1265 \\ Military Trail, Toronto, Ontario, Canada M1C 1A4. \\ ${ }^{*}$ To whom correspondence should be addressed, E-mail: frank.wania@utoronto.ca
}

\section{Table of Content}

Table S1 Dates, meteorological conditions and specific snow surface areas for the five sampled snowfall events.

Figure S1 Time course of the daily mean, maximum and minimum temperature during the ageing of snow falling during five events at Turkey Lakes in the winter of $1999 / 2000$.

Table S2 Instrument detection limit for OCPs and PCBs in picograms.

Table S3 Concentration of organochlorine pesticides and chlorobenzenes in snowmelt water in pg/L SWE (mean and relative standard deviation of 3 replicates).

Table S4 Concentration of total polychlorinated biphenyls in snowmelt water in ng/L SWE (mean and relative standard deviation of 3 replicates) and homologue composition.

Figure S2 Concentration changes of polychlorinated biphenyl congeners during the ageing of four snowpacks as a function of the congeners' snow-air partition coefficients $K_{\mathrm{SA}}$ adjusted to the mean temperature during the ageing period. 
Table S1 Dates, meteorological conditions and specific snow surface areas for the five sampled snowfall events.

\begin{tabular}{|c|c|c|c|c|c|}
\hline & Event 1 & Event 2 & Event 3 & Event 4 & Event 5 \\
\hline Day of first sampling & 23/12/99 & $20 / 1 / 00$ & $24 / 1 / 00$ & $9 / 2 / 00$ & $16 / 3 / 00$ \\
\hline Day of second sampling & $5 / 1 / 00$ & $31 / 1 / 00$ & $31 / 1 / 00$ & $16 / 2 / 00$ & $20 / 3 / 00$ \\
\hline Length of snow ageing in days & 13 & 11 & 7 & 7 & 4 \\
\hline $\begin{array}{l}\text { Wind direction and speed during } \\
\text { snowfall }\end{array}$ & $\mathrm{NE}, 1.1 \mathrm{~m} / \mathrm{s}$ & $\mathrm{N}, 2.1 \mathrm{~m} / \mathrm{s}$ & $\mathrm{W}, 1.6 \mathrm{~m} / \mathrm{s}$ & $\mathrm{NW}, 3.2 \mathrm{~m} / \mathrm{s}$ & $\mathrm{N}, 1.8 \mathrm{~m} / \mathrm{s}$ \\
\hline Temperature during snowfall in ${ }^{\circ} \mathrm{C}$ & $\begin{array}{l}-14.2 \\
(-9.2 \text { to }-18.2)\end{array}$ & $\begin{array}{l}-19.5 \\
(-12.3 \text { to }-25.0)\end{array}$ & $\begin{array}{l}-10.7 \\
(-8.0 \text { to }-13.5)\end{array}$ & $\begin{array}{l}-4.1 \\
(-9.5 \text { to }-0.4)\end{array}$ & $\begin{array}{l}-8.1 \\
(-13.6 \text { to }-2.6)\end{array}$ \\
\hline $\begin{array}{l}\text { Mean and range of daily mean wind } \\
\text { speed during ageing }\end{array}$ & $2.3(0.7$ to 4.6$)$ & 1.5 (0.6 to 2.5$)$ & $1.4(0.6$ to 2.5$)$ & $2.2(1.0$ to 3.2$)$ & $2.2(1.8$ to 3.0$)$ \\
\hline SWE of snowfall event in mm & $9.6 \mathrm{~mm}$ & $6.6 \mathrm{~mm}^{\mathrm{a}}$ & $7.4 \mathrm{~mm}$ & $13.9 \mathrm{~mm}$ & $11.2 \mathrm{~mm}$ \\
\hline $\begin{array}{l}\text { Range of daily mean temperature during } \\
\text { ageing in }{ }^{\circ} \mathrm{C}\end{array}$ & -16.3 to -2.5 & -22 to -3.6 & -19.3 to -3.6 & -14 to -4 & -7.6 to +2.6 \\
\hline $\begin{array}{l}\text { Overall range of temperature during } \\
\text { ageing in }{ }^{\circ} \mathrm{C}\end{array}$ & -21.7 to +0.7 & -29.1 to +1.7 & -23.1 to +1.7 & -19.6 to -0.4 & -14.2 to +5.0 \\
\hline Mean temperature during ageing in ${ }^{\circ} \mathrm{C}$ & -10.9 & -12.8 & -10.0 & -9.7 & -3.0 \\
\hline $\begin{array}{l}\text { Specific surface area immediately after } \\
\text { snowfall in } \mathrm{cm}^{2} / \mathrm{g}\end{array}$ & $\begin{array}{l}1223 \pm 38 \\
(\mathrm{n}=3)\end{array}$ & $\begin{array}{l}1332 \pm 203 \\
(\mathrm{n}=4)\end{array}$ & $\begin{array}{l}1220 \pm 51 \\
(\mathrm{n}=3)\end{array}$ & $\begin{array}{l}1014 \pm 11 \\
(\mathrm{n}=3)\end{array}$ & $\begin{array}{l}1115 \pm 135 \\
(\mathrm{n}=3)\end{array}$ \\
\hline $\begin{array}{l}\text { Specific surface area after ageing in } \\
\mathrm{cm}^{2} / \mathrm{g}\end{array}$ & $\begin{array}{l}685 \pm 217 \\
(\mathrm{n}=3)\end{array}$ & $\begin{array}{l}595 \pm 19 \\
(n=3)\end{array}$ & $\begin{array}{l}776 \pm 54 \\
(\mathrm{n}=3)\end{array}$ & $\begin{array}{l}559 \pm 19 \\
(n=3)\end{array}$ & $\begin{array}{l}520 \pm 50 \\
(n=3)\end{array}$ \\
\hline $\begin{array}{l}\text { Snowmelt water volume extracted before } \\
\text { ageing in L }\end{array}$ & $\begin{array}{l}17.0 \pm 0.3 \\
(\mathrm{n}=3)\end{array}$ & $\begin{array}{l}5.1 \pm 0.1 \\
(\mathrm{n}=3)\end{array}$ & $\begin{array}{l}7.1 \pm 0.2 \\
(\mathrm{n}=3)\end{array}$ & $\begin{array}{l}6.2 \pm 0.3 \\
(\mathrm{n}=3)\end{array}$ & $\begin{array}{l}9.5 \pm 0.1 \\
(n=3)\end{array}$ \\
\hline $\begin{array}{l}\text { Snowmelt water volume extracted after } \\
\text { ageing in L }\end{array}$ & $\begin{array}{l}23.4 \pm 1.1 \\
(\mathrm{n}=3)^{\mathrm{b}}\end{array}$ & $\begin{array}{l}4.8 \pm 0.1 \\
(\mathrm{n}=3)\end{array}$ & $\begin{array}{l}6.4 \pm 0.3 \\
(\mathrm{n}=3)\end{array}$ & $\begin{array}{l}6.1 \pm 0.4 \\
(\mathrm{n}=3)\end{array}$ & $\begin{array}{l}11.9 \pm 1.7^{\mathrm{c}} \\
(\mathrm{n}=3)\end{array}$ \\
\hline
\end{tabular}

${ }^{a}$ sum of the precipitation falling from Jan. 18 to 20, 2000, ${ }^{b}$ snow meltwater volume increased during ageing, likely because of blowing snow, ${ }^{c}$ snow meltwater volume increased during ageing, likely because of rain falling onto snowpack. 


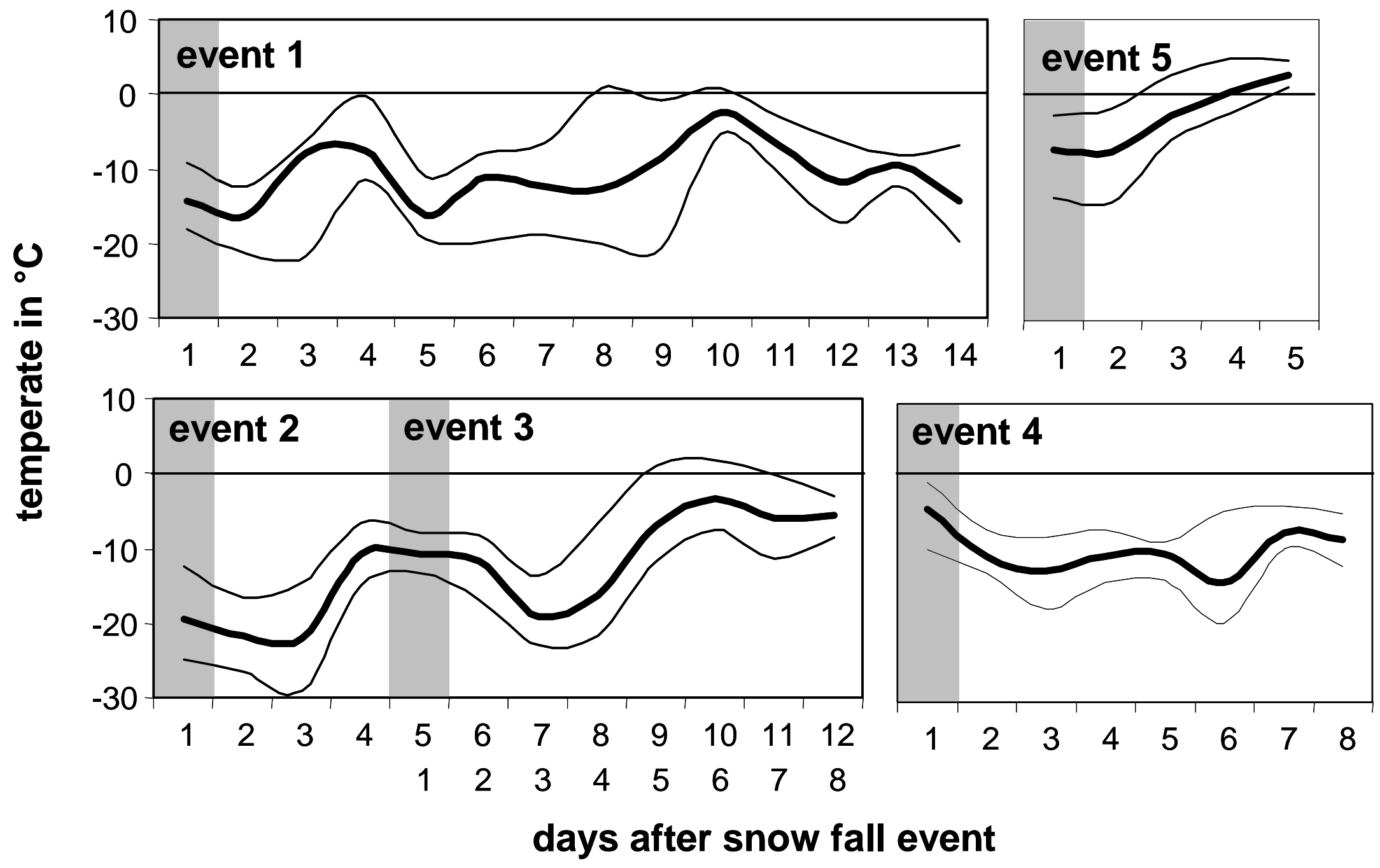

Figure S1 Time course of the daily mean, maximum and minimum temperature during the ageing of snow falling during five events at Turkey Lakes in the winter of 1999/2000. 
Table S2 Instrument detection limit for organochlorine pesticides and polychlorinated biphenyls in picograms. Method detection limits vary between samples because of different sample volume

\begin{tabular}{|c|c|c|c|c|c|c|c|c|c|}
\hline \multicolumn{2}{|l|}{ OCPs } & \multicolumn{2}{|l|}{ PCB } & \multicolumn{2}{|l|}{ PCB } & \multicolumn{2}{|l|}{ PCB } & \multicolumn{2}{|l|}{ PCB } \\
\hline$\alpha-H C H$ & 0.20 & 3 & 0.09 & 46 & 0.02 & 110 & 0.03 & 177 & 0.01 \\
\hline$\beta-H C H$ & 0.20 & $4+10$ & 0.05 & 47 & 0.04 & 114 & 0.02 & 178 & 0.02 \\
\hline \multirow[t]{2}{*}{$\gamma-\mathrm{HCH}$} & 0.30 & $5+8$ & 0.11 & 48 & 0.04 & 118 & 0.02 & 180 & 0.03 \\
\hline & & 6 & 0.03 & 52 & 0.02 & 119 & 0.01 & $182+187$ & 0.03 \\
\hline dieldrin & 0.38 & 7 & 0.04 & $56+60$ & 0.04 & $124+135+144$ & 0.02 & 183 & 0.02 \\
\hline endrin & 0.10 & $12+13$ & 0.02 & 63 & 0.02 & 128 & 0.03 & 185 & 0.01 \\
\hline heptachlor epoxide & 0.06 & 16 & 0.03 & 66 & 0.03 & 129 & 0.01 & 189 & 0.04 \\
\hline$\alpha$-chlordane & 0.03 & 17 & 0.03 & 70 & 0.03 & 130 & 0.01 & 190 & 0.01 \\
\hline$\gamma$-chlordans & 0.04 & 18 & 0.05 & 74 & 0.02 & $134+143$ & 0.03 & 191 & 0.04 \\
\hline$\alpha$-endosulfan & 0.15 & 19 & 0.02 & $81+87$ & 0.02 & 136 & 0.01 & 193 & 0.01 \\
\hline$\beta$-endosulfan & 0.04 & $21+33+53$ & 0.05 & 82 & 0.06 & 137 & 0.02 & 194 & 0.01 \\
\hline \multirow[t]{2}{*}{ Trans-nonachlor } & 0.04 & 22 & 0.04 & 83 & 0.02 & $138+163$ & 0.03 & 195 & 0.05 \\
\hline & & $24+27$ & 0.02 & 84 & 0.02 & 141 & 0.01 & $196+203$ & 0.07 \\
\hline pp'-DDE & 0.02 & 25 & 0.02 & 85 & 0.03 & 146 & 0.04 & 197 & 0.05 \\
\hline pp'-DDT & 0.05 & 26 & 0.04 & 89 & 0.01 & 151 & 0.02 & 198 & 0.03 \\
\hline \multirow[t]{2}{*}{ Pp'-DDD } & 0.03 & 28 & 0.05 & 91 & 0.02 & 153 & 0.03 & 199 & 0.02 \\
\hline & & 29 & 0.04 & 92 & 0.08 & $156+171$ & 0.03 & 201 & 0.04 \\
\hline HCB & 0.04 & 31 & 0.02 & 95 & 0.03 & 157 & 0.05 & 202 & 0.01 \\
\hline aldrin & 0.04 & 32 & 0.02 & 97 & 0.02 & 158 & 0.01 & 205 & 0.02 \\
\hline heptachlor & 0.08 & 40 & 0.02 & 99 & 0.02 & 167 & 0.01 & 206 & 0.04 \\
\hline methoxychlor & 0.09 & $41+64+71$ & 0.03 & 100 & 0.01 & 170 & 0.02 & 207 & 0.01 \\
\hline \multirow[t]{4}{*}{ mirex } & 0.30 & 42 & 0.03 & 101 & 0.02 & 172 & 0.02 & 208 & 0.01 \\
\hline & & $43+49$ & 0.02 & 103 & 0.04 & 174 & 0.01 & 209 & 0.02 \\
\hline & & 44 & 0.02 & $105+132$ & 0.03 & 175 & 0.03 & & \\
\hline & & 45 & 0.02 & $107+142$ & 0.05 & 176 & 0.01 & & \\
\hline
\end{tabular}


Table S3 Concentration of organochlorine pesticides and chlorobenzenes in snowmelt water in pg/L SWE (mean and relative standard deviation of 3 replicates).

\begin{tabular}{|c|c|c|c|c|c|c|c|c|c|c|}
\hline & $\begin{array}{l}\text { Event } 1 \\
\text { fresh snow }\end{array}$ & $\begin{array}{l}\text { Event } 1 \\
\text { aged snow }\end{array}$ & $\begin{array}{l}\text { Event } 2 \\
\text { fresh snow }\end{array}$ & $\begin{array}{l}\text { Event } 2 \\
\text { aged snow }\end{array}$ & $\begin{array}{l}\text { Event } 3 \\
\text { fresh snow }\end{array}$ & $\begin{array}{l}\text { Event } 3 \\
\text { aged snow }\end{array}$ & $\begin{array}{l}\text { Event } 4 \\
\text { fresh snow }\end{array}$ & $\begin{array}{l}\text { Event } 4 \\
\text { aged snow }\end{array}$ & $\begin{array}{l}\text { Event } 5 \\
\text { fresh snow }\end{array}$ & $\begin{array}{l}\text { Event } 5 \\
\text { aged snow }\end{array}$ \\
\hline$\alpha-\mathrm{HCH}$ & 979 (23\%) & 133 (17\%) & $122(9 \%)$ & $54(6 \%)$ & $135(8 \%)$ & $54(17 \%)$ & $58(22 \%)$ & $61(29 \%)$ & 73 (13\%) & $638(65 \%)$ \\
\hline$\gamma-\mathrm{HCH}$ & 435 (19\%) & 118 (15\%) & 126 (16\%) & 67 (17\%) & 108 (15\%) & 65 (21\%) & 177 (37\%) & 118 (21\%) & 113 (8.5\%) & 302 (54\%) \\
\hline Dieldrin & $198(16 \%)$ & 116 (10\%) & 208 (58\%) & $102(18 \%)$ & 117 (18\%) & 77 (17\%) & $222(42 \%)$ & 139 (19\%) & 84 (5.1\%) & $161(42 \%)$ \\
\hline endosulfan-I & $181(24 \%)$ & 76 (7.7\%) & 73 (28\%) & $52(11 \%)$ & $59(45 \%)$ & 32 (75\%) & 31 (71\%) & $64(7 \%)$ & 56 (8.3\%) & $122(41 \%)$ \\
\hline endosulfan-II & $11(23 \%)$ & $10(18 \%)$ & $19(17 \%)$ & 23 (18\%) & $6.1(86 \%)$ & $8.4(25 \%)$ & 22 (19\%) & 35 (14\%) & 22 (39\%) & $22(14 \%)$ \\
\hline heptachlor epox. & $60(23 \%)$ & $21(20 \%)$ & 29 (16\%) & 17 (23\%) & $32(20 \%)$ & 22 (19\%) & 30 (16\%) & 26 (61\%) & 25 (12\%) & 42 (61\%) \\
\hline cis-chlordane & 22 (60\%) & $16(16 \%)$ & $22(25 \%)$ & 16 (17\%) & 7.1(141\%) & $8.1(34 \%)$ & 20 (124\%) & $13(43 \%)$ & $7.3(73 \%)$ & 19 (53\%) \\
\hline trans-chlordane & $26(73 \%)$ & $9.2(2 \%)$ & 19 (54\%) & $14(41 \%)$ & $19(71 \%)$ & $14(24 \%)$ & 15 (16\%) & 32 (46\%) & 35 (15\%) & 54 (34\%) \\
\hline trans-nonachlor & $18(7 \%)$ & 16 (35\%) & 25 (43\%) & $10(18 \%)$ & $21(35 \%)$ & $15(26 \%)$ & $20(11 \%)$ & $12(17 \%)$ & $14(71 \%)$ & 17 (45\%) \\
\hline oxychlordane & & 10 (73\%) & & $15(71 \%)$ & & $11(80 \%)$ & & & & 50 (56\%) \\
\hline methoxychlor & & & & 41 (100\%) & & 42 (35\%) & & & $11(14 \%)$ & \\
\hline pp'-DDE & $8(71 \%)$ & $11(16 \%)$ & $8.0(74 \%)$ & 4.7 (141\%) & $4.0(141 \%)$ & 7.5 (43\%) & $2.9(141 \%)$ & $14(40 \%)$ & 8.7 (79\%) & 17 (11\%) \\
\hline pp'-DDT & 36 (19\%) & 23 (6.3\%) & 40 (35\%) & 21 (28\%) & 21 (88\%) & $10(24 \%)$ & 23 (46\%) & 42 (36\%) & 29 (49\%) & $57(6 \%)$ \\
\hline pp'-DDD & & $2.8(20 \%)$ & & $6.8(141 \%)$ & & $17(8 \%)$ & $23(22 \%)$ & 36 (100\%) & $2.8(85 \%)$ & 4.6 (71\%) \\
\hline op'-DDE & $5.2(27 \%)$ & $3.2(77 \%)$ & & & $4.1(141 \%)$ & 8.5 (29\%) & & $4.1(141 \%)$ & 4.5 (89\%) & $6.0(71 \%)$ \\
\hline op'-DDT & 12 (33\%) & 9.3 (37\%) & 10 (71\%) & $2.3(141 \%)$ & & 8.3 (16\%) & & $6.1(75 \%)$ & & $12(71 \%)$ \\
\hline op'-DDD & & 163 (8.6\%) & & 16 (72\%) & 20 (71\%) & & & $11(72 \%)$ & 20 (79\%) & 324 (61\%) \\
\hline 135-ТСВ & & & & 7.4 (141\%) & & & & $8.4(141 \%)$ & & \\
\hline 124-ТСВ & 38 (25\%) & 28 (19\%) & 179 (19\%) & 149 (7\%) & 116 (26\%) & $85(27 \%)$ & $73(2 \%)$ & $62(24 \%)$ & 33 (76\%) & 32 (31\%) \\
\hline 123-ТСВ & & & $10(71 \%)$ & 16 (141\%) & & 12 (79\%) & & & 13 (141\%) & 4.7 (141\%) \\
\hline 1234-ТЕCВ & 4.2 (53\%) & 3 (49\%) & 12 (12\%) & $4.6(141 \%)$ & 3.7 (141\%) & 1.9 (141\%) & $3.9(74 \%)$ & $5.3(72 \%)$ & $642(71 \%)$ & $2.7(17 \%)$ \\
\hline PeCB & 19 (49\%) & 10 (25\%) & 26 (19\%) & 16 (31\%) & $19(20 \%)$ & $11(9 \%)$ & 12 (13\%) & $14(6 \%)$ & 16 (72\%) & 20 (20\%) \\
\hline НСB & 11 (9\%) & 8 (14\%) & $16(6 \%)$ & $12(10 \%)$ & 24 (70\%) & $6.4(5 \%)$ & 12 (23\%) & $10(8 \%)$ & 12 (46\%) & 15 (9\%) \\
\hline
\end{tabular}


Table S4 Concentration of total polychlorinated biphenyls in snowmelt water in ng/L SWE (mean and relative standard deviation of 3 replicates) and homologue composition.

\begin{tabular}{|c|c|c|c|c|c|c|c|c|c|c|}
\hline & $\begin{array}{l}\text { Event } 1 \\
\text { fresh } \\
\text { snow }\end{array}$ & $\begin{array}{l}\text { Event } 1 \\
\text { aged } \\
\text { snow }\end{array}$ & $\begin{array}{l}\text { Event } 2 \\
\text { fresh } \\
\text { snow }\end{array}$ & $\begin{array}{l}\text { Event } 2 \\
\text { aged } \\
\text { snow }\end{array}$ & $\begin{array}{l}\text { Event } 3 \\
\text { fresh } \\
\text { snow }\end{array}$ & $\begin{array}{l}\text { Event } 3 \\
\text { aged } \\
\text { snow }\end{array}$ & $\begin{array}{l}\text { Event } 4 \\
\text { fresh } \\
\text { snow }\end{array}$ & $\begin{array}{l}\text { Event } 4 \\
\text { aged } \\
\text { snow }\end{array}$ & $\begin{array}{l}\text { Event } 5 \\
\text { fresh } \\
\text { snow }\end{array}$ & $\begin{array}{l}\text { Event } 5 \\
\text { aged } \\
\text { snow }\end{array}$ \\
\hline Sum PCBs 1994+ & $\begin{array}{l}4.7 \\
(43 \%)\end{array}$ & $\begin{array}{l}\mathbf{1 . 8} \\
(27 \%)\end{array}$ & $\begin{array}{l}\mathbf{4 . 0} \\
(36 \%)\end{array}$ & $\begin{array}{l}2.1 \\
(13 \%)\end{array}$ & $\begin{array}{l}3.0 \\
(41 \%)\end{array}$ & $\begin{array}{l}3.3 \\
(22 \%)\end{array}$ & $\begin{array}{l}2.1 \\
(24 \%)\end{array}$ & $\begin{array}{l}2.1 \\
(24 \%)\end{array}$ & $\begin{array}{l}1.3 \\
(21 \%)\end{array}$ & $\begin{array}{l}\mathbf{5 . 0} \\
(42 \%)\end{array}$ \\
\hline DichloroCBs & $3.7 \%$ & $2.4 \%$ & $4.6 \%$ & $5.8 \%$ & $7.9 \%$ & $5.9 \%$ & $5.7 \%$ & $6.2 \%$ & $4.8 \%$ & $2.9 \%$ \\
\hline TrichloroCBs & $18 \%$ & $13 \%$ & $14 \%$ & $15 \%$ & $17 \%$ & $36 \%$ & $20 \%$ & $20 \%$ & $19 \%$ & $18 \%$ \\
\hline TetrachloroCBs & $39 \%$ & $36 \%$ & $39 \%$ & $33 \%$ & $38 \%$ & $34 \%$ & $38 \%$ & $37 \%$ & $37 \%$ & $34 \%$ \\
\hline PentachloroCBs & $26 \%$ & $29 \%$ & $25 \%$ & $29 \%$ & $21 \%$ & $17 \%$ & $23 \%$ & $23 \%$ & $24 \%$ & $27 \%$ \\
\hline HexachloroCBs & $11 \%$ & $14 \%$ & $13 \%$ & $14 \%$ & $12 \%$ & $6.1 \%$ & $12 \%$ & $9.5 \%$ & $8.7 \%$ & $13 \%$ \\
\hline HeptachloroCBs & $1.7 \%$ & $2.8 \%$ & $1.4 \%$ & $1.3 \%$ & $3.1 \%$ & $0.42 \%$ & $1.0 \%$ & $1.4 \%$ & $3.1 \%$ & $1.6 \%$ \\
\hline OctachloroCBs & $0.29 \%$ & $0.55 \%$ & $0.70 \%$ & $0.55 \%$ & $0.68 \%$ & $0.21 \%$ & $0.56 \%$ & $0.38 \%$ & $0.19 \%$ & $0.10 \%$ \\
\hline
\end{tabular}



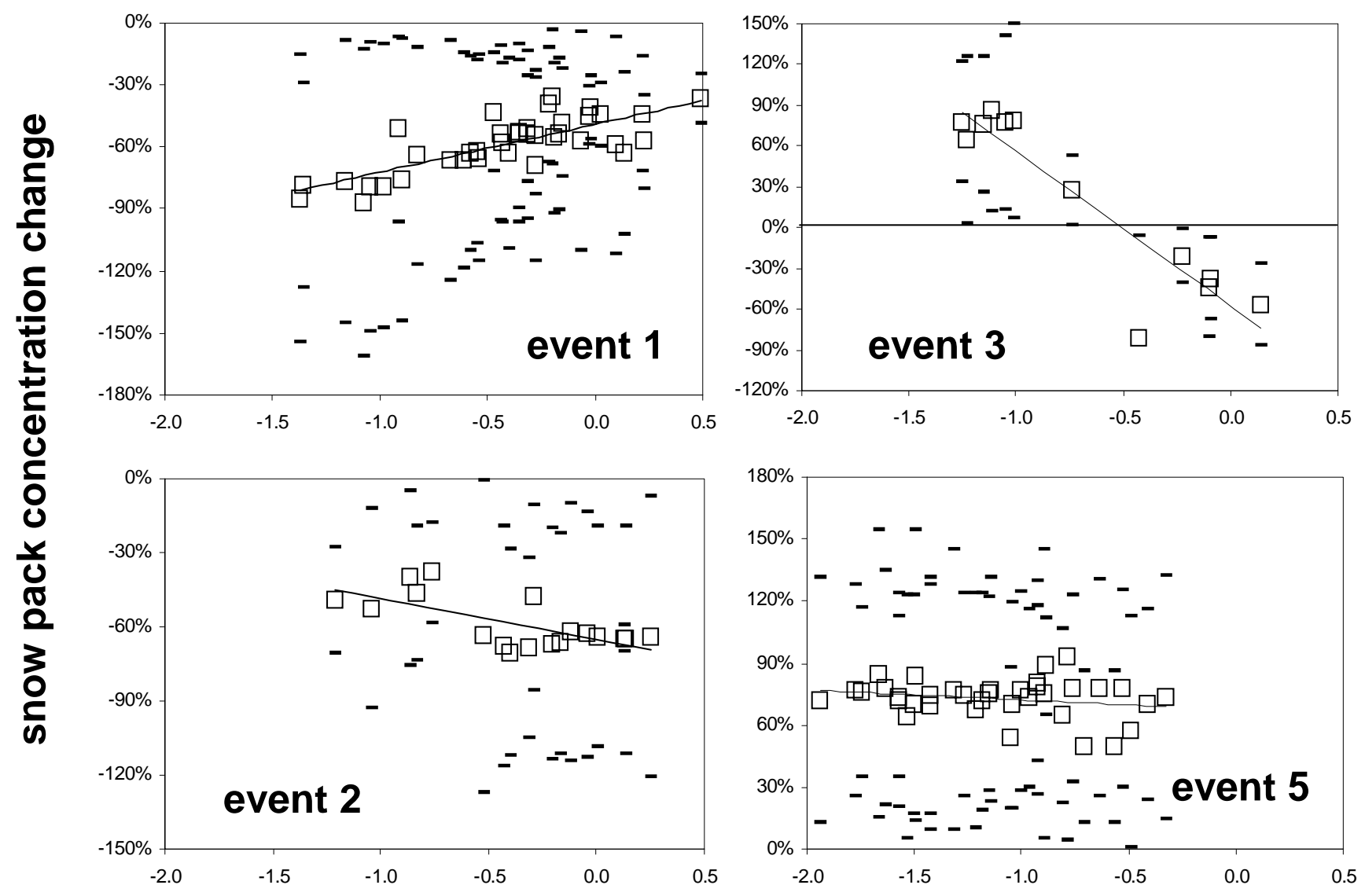

\section{$\log \left(\mathrm{K}_{\mathrm{SA}} \mathrm{Im}\right)$}

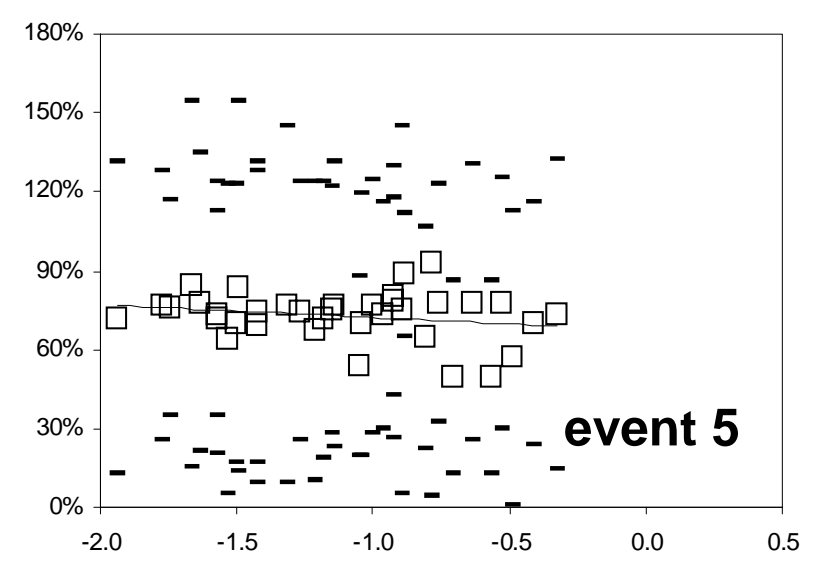

$\log \left(\mathrm{K}_{\mathrm{SA}} / \mathrm{m}\right)$

Figure S2 Concentration changes of polychlorinated biphenyl congeners during the ageing of four snowpacks as a function of the congeners' snow-air partition coefficients $K_{\mathrm{SA}}$ adjusted to the mean temperature during the ageing period. Concentrations of PCBs during event 4 did not change significantly and are not displayed. Negative values indicate loss of chemical during ageing, positive values indicate a concentration increase. The square marks the mean, and the bars the mean plus/minus one standard deviation, calculated by error propagation from the standard deviation of the snow concentrations. 